1 Systemic Effects of Optos versus Indirect Ophthalmoscopy for Retinopathy of Prematurity Screening

3

4 Timothy H.M. Fung, MBChB, ${ }^{1}$ Janine Abramson, ${ }^{1,2}$ Shalini Ojha, PhD, ${ }^{1,2}$ Roger 5 Holden, MBBS, 1

6

$7 \quad{ }^{1}$ Royal Derby Hospital, Uttoxeter Road, Derby, DE22 3NE, United Kingdom

$8{ }^{2}$ School of Medicine, University of Nottingham, Nottingham, NG7 2UH, United

9 Kingdom

11 To be presented in part at: Association for Research in Vision and 12 Ophthalmology Annual Meeting May 2018, Honolulu, Hawaii.

14 Financial support: We thank Optos PLC (Scotland, Dunfermline, United Kingdom) 15 for providing us with $£ 2000$ to cover the costs of conducting the study. Optos 16 PLC had no part in the design or conduct of this research. Optos PLC also had no 17 part in the analysis of the data or the presentation of the findings.

19 No conflicting relationship exists for any author.

21 Correspondence to Dr Timothy HM Fung, Department of Ophthalmology, Royal 22 Derby Hospital, Derby, DE22 3NE, United Kingdom. Email: timothyfung@doctors.org.uk 


\section{Unstructured abstract}

27 A prospective randomised cross over study demonstrating for the first time that 28 bedside retinopathy of prematurity screening is more stressful with the Optos

29 California than with conventional binocular indirect ophthalmoscopy.

30

31

32

33

34

35

36

37

38

39

40

41

42

43

44

45

46

47

48

49 
50 Many regions of the world have a relative shortage of ophthalmologists trained

51 and willing to screen for retinopathy of prematurity (ROP). ${ }^{1}$ As a result, many

52 regions have turned to retinal imaging and telemedicine. ${ }^{2}$ Optos ultra-widefield

53 retinal imaging (Optos PLC, Dunfermline, UK) has recently been used as a

54 screening tool for ROP.3,4 However, there is no data available regarding the

55 systemic effects or stress induced by an ROP screening examination with the

56 Optos. In this prospective randomised cross over study we determined the

57 effects of Optos ultra-widefield screening examination on cardiorespiratory

58 indices, as a measure of stress, and compared the stress response to that of

59 conventional binocular indirect ophthalmoscopy (BIO) examination. The East

60 Midlands Leicester South Research Ethics Committee granted permission to

61 undertake the study. Written informed consents were obtained from the

62 mothers of all infants involved in the study. The study adhered to the tenets of

63 the Declaration of Helsinki.

64 Any infants born at $\leq 32$ week's gestation or weighing $\leq 1500$ grams at birth were

65 included. Each infant underwent bedside examination with the Optos California

66 (Optos) and BIO for all of its scheduled screening sessions. The order of the

67 examination methods performed was determined by randomisation via sealed

68 envelopes prepared remotely by a member of the team not involved in the

69 recruitment or screening of infants. Mydriasis was achieved with cyclopentolate

$70 \quad 0.5 \%$ and phenylephrine $2.5 \%$ instilled in each eye at 30 minutes prior to

71 examination commencing. Optos examination began with the instillation of

72 proxymetacaine $0.5 \%$ into each eye followed by placement of a speculum in the

73 right eye. The infant was gently lifted out of the incubator or cot and held upright

74 to the Optos. Pseudocolour retinal images were acquired and the speculum was 
then transferred to the fellow eye. Once images were captured from the fellow eye, the speculum was removed and the examination was deemed complete. BIO examination began with the instillation of proxymetacaine $0.5 \%$ into each eye followed by insertion of a speculum in the right eye. A 28-diopter lens was used together with an indirect ophthalmoscope to examine the retina of the infant in the supine position. The speculum was then transferred to the fellow eye and the retinal examination was repeated. Scleral indentation was not used. Once the examination was completed in the fellow eye, the speculum was removed and the examination was deemed complete. One neonatology nurse recorded observations of $\mathrm{SaO}_{2}$, $\mathrm{HR}$ in beats per minute, and respiratory rate (RR) in breaths per minute on data collection forms for each examination method. $\mathrm{SaO}_{2}$ and HR were measured using pulse oximetry, whereas the RR was recorded manually. All of the above cardiorespiratory indices were recorded immediately before instillation of topical anesthesia into both eyes (baseline values), immediately after insertion of the speculum into the first eye to be examined, immediately after the start and completion of the examination, and 10 minutes after the completion of the examination. The examination time, measured from the time of instillation of anesthetic drops to the removal of the speculum from the second eye examined, was recorded for each examination method.

The results of 50 screening examinations with the Optos and BIO on 26 infants (16 males and 10 females) were analysed. Of the 26 infants screened, two infants had stage 3 ROP and one infant had stage 1 ROP. The rest of the infants had no ROP. The mean gestational age was 27.9 weeks (standard deviation [SD], 2.2; range, 24-34) and the mean birth weight was 1135 g (SD, 375.8; range, 6842110). The mean postnatal age at the time of examination was 34.5 weeks (SD, 
2.2; range, 28-39). There was a median of two screening examinations per infant

101 (range, 1-4). Of the 50 screening examinations, 50\% were randomised to have

102 the ROP screening examination starting with the Optos followed by BIO.

103 The cardiorespiratory indices over time for the two examination methods are 104 shown in Figure 1. Using the paired T-test, none of the three cardiorespiratory 105 indices varied significantly at baseline between the Optos and BIO. Some infants 106 in the study underwent more than one screening examination with the Optos 107 and BIO. Additionally, some infants underwent repeated measurements from 108 each screening examination, due to both multiple timepoints and measurements 109 by two examination methods. To account for this, a three level model linear 110 regression analysis was used with individual measurements nested within 111 individual screening examinations, which in turn was contained within infants.

112 By using multilevel linear regression analysis, we found no significant difference 113 in HR or RR between the two examination methods $(P=0.49$ and $P=0.56$, 114 respectively). However, a significant difference in $\mathrm{SaO}_{2}(P=0.04)$ between the 115 examination methods was found. The Optos examination method gave lower $116 \mathrm{SaO}_{2}$ values, with values being, on average, $0.8 \%$ lower for the Optos group than 117 for the BIO group. Handling of the infants required to position for image capture 118 with the Optos may explain this finding. Mean examination times were 3.0 119 minutes (SD, 0.7) in the Optos group and 1.8 minutes (SD, 0.5) in the BIO group $120(\mathrm{P}<0.001)$. The longer duration of screening examination with the Optos might 121 be a contributing factor to the lower $\mathrm{SaO}_{2}$ levels found when compared to $\mathrm{BIO}$ 122 examinations. No episodes of clinically significant bradycardia (HR $<100$ beats 123 per minute) or respiratory distress ( $R R<30$ breaths per minute) occurred 124 during or after any screening examinations with the Optos or BIO. Of the 50 
125 screening examinations, $5(10 \%)$ in the Optos group and only $1(2 \%)$ in the BIO

126 group developed clinically significant oxygen desaturation $\left(\mathrm{SaO}_{2}<85 \%\right)$ during

127 examination, precluding statistical analysis. No additional supplemental oxygen

128 administration to the infants during these screening examinations was required

129 as $\mathrm{SaO}_{2}$ levels increased spontaneously or with stimulation. All episodes of

130 clinically significant oxygen desaturation occurred for screening examinations

131 performed in infants in the intensive care unit of the neonatology department.

132 Limitations of our study include firstly the small number of infants in a single

133 center. Further studies with larger number of infants are required to verify our

134 findings. Secondly, our institution provides only level 2 neonatal care and our

135 study population may not be generalisable to sicker infants with more co-

136 morbidities. Thirdly, the majority of the infants screened in this study had no

137 ROP. Examination with BIO in this study may therefore have been less extensive

138 or caused less distress to the infants than would normally be experienced in

139 neonatology units that have infants with more severe forms of ROP. Finally,

140 further studies that measure both the physiological and behavioural responses of

141 stress are required to determine the complete stress response of infants to

142 screening with the Optos.

143 In summary, the statistical difference in $\mathrm{SaO}_{2}$ and the difference in the number of

144 clinically significant oxygen desaturations found may indicate that ROP

145 screening is more stressful with the Optos than with BIO. Careful handling and

146 close monitoring of $\mathrm{SaO}_{2}$ should be performed when the Optos is used for

147 bedside screening. Greater attention should be paid to the sicker infants in the

148 intensive care unit who seem to be the most vulnerable to clinically significant

149 oxygen desaturations with the Optos examination. 
151 References

152 1. Gilbert C, Rahi J, Eckstein M, et al. Retinopathy of prematurity in middle153 income countries. Lancet 1997;350:12-4.

154 2. Chiang MF, Wang L, Busuioc M, et al. Telemedical retinopathy of prematurity 155 diagnosis: accuracy, reliability, and image quality. Arch Ophthalmol $156 \quad 2007 ; 125: 1531-8$.

157 3. Patel CK, Fung THM, Muqit MM, et al. Non-contact ultra-widefield imaging of 158 retinopathy of prematurity using the Optos dual wave-length scanning laser 159 ophthalmoscope. Eye (Lond) 2013;27:589-96.

160 4. Arnold RW, Grendahl RL, Winkle K, et al. Outpatient, wide-field, digital 161 imaging of infants with retinopathy of prematurity. Ophthalmic Surg Lasers $162 \quad$ Imaging 2017;48:494-7.

163 5. Laws De, Morton C, Weindling M, et al. Systemic effects of screening for 164 retinopathy of prematurity. Br J Ophthalmol 1996;80:425-8. 

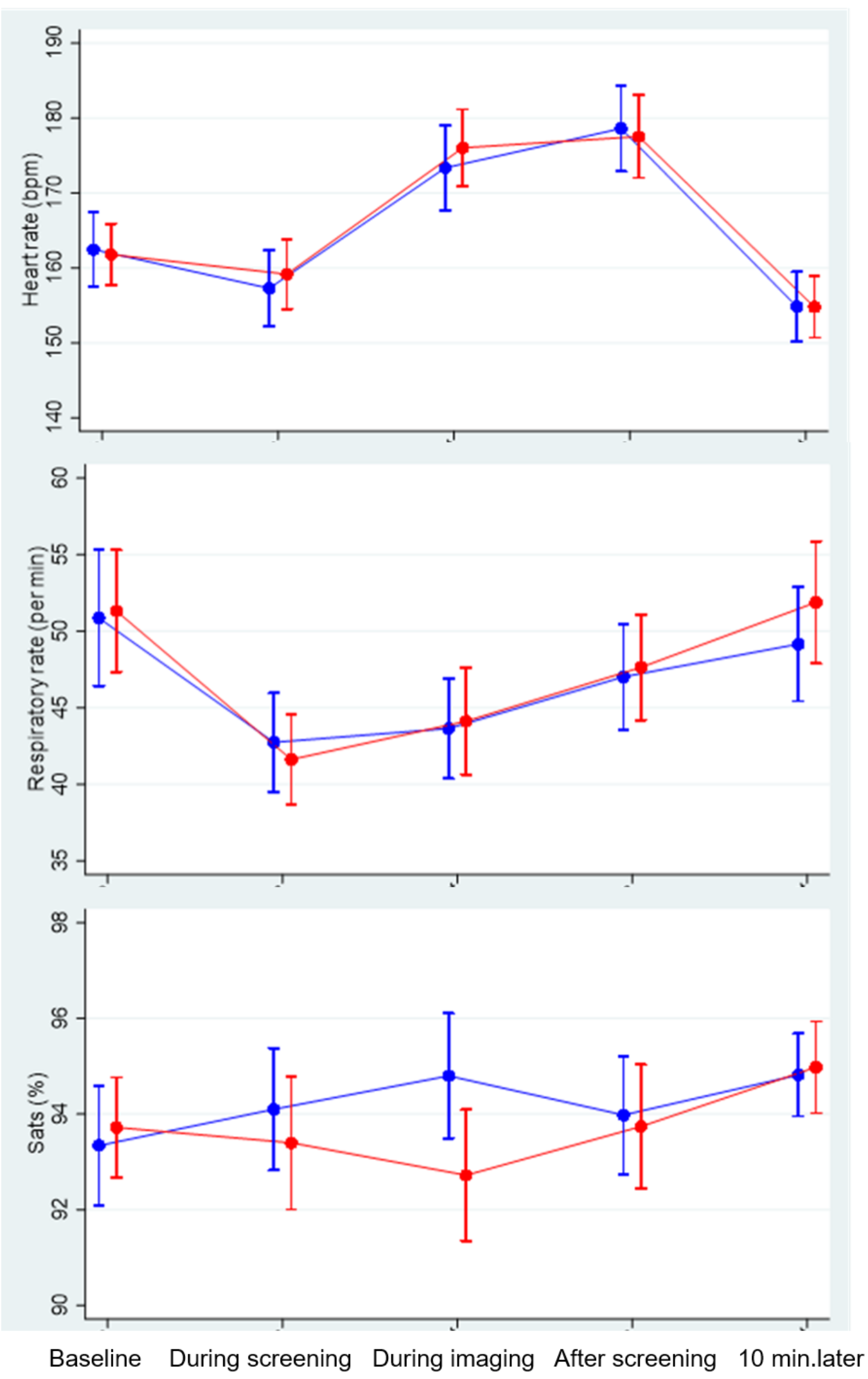

167 Figure 1. Cardiorespiratory indices during OPTOS and BIO examinations

168 Recordings during BIO are represented in blue and during OPTOS in red.

169 bpm, beats per minute; Sats, oxygen saturation level 